\title{
PILAR: a Federation of VISIR Remote Laboratory Systems for Educational Open Activities
}

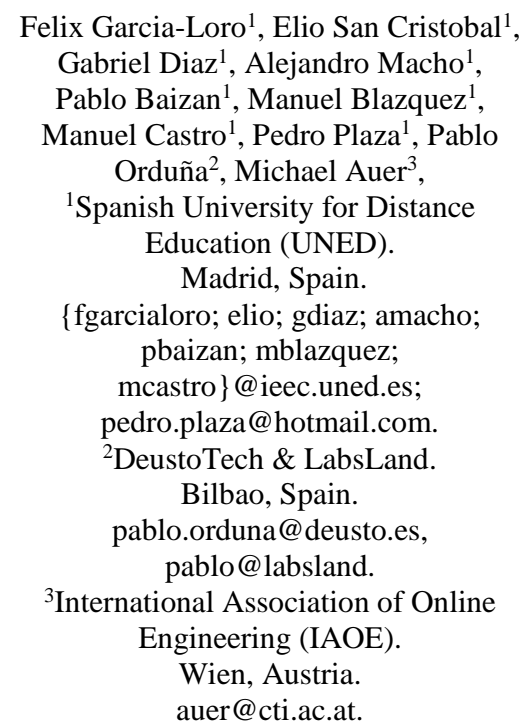

Felix Garcia-Loro $^{1}$, Elio San Cristobal ${ }^{1}$, Gabriel Diaz ${ }^{1}$, Alejandro Macho ${ }^{1}$, Pablo Baizan ${ }^{1}$, Manuel Blazquez ${ }^{1}$, Manuel Castro ${ }^{1}$, Pedro Plaza ${ }^{1}$, Pablo Orduña ${ }^{2}$, Michael Auer ${ }^{3}$,

${ }^{1}$ Spanish University for Distance ducation (UNED) Madrid, Spain pbaizan; mblazquez; mcastro\}@ieec.uned.es; pedro.plaza@hotmail.com. lo.orduna@deusto.es,

Wien, Austria. auer@cti.ac.at.

\author{
Wlodek Kulesza ${ }^{4}$, Ingvar Gustavsson ${ }^{4}$, \\ Kristian Nilsson ${ }^{4}$, Andre Fidalgo ${ }^{5}$, \\ Gustavo Alves ${ }^{5}$, Arcelina Marques ${ }^{5}$, \\ Unai Hernandez-Jayo ${ }^{6}$, Javier Garcia- \\ Zubia $^{6}$ \\ ${ }^{4}$ Blekinge Institute of Technology \\ (BTH). \\ Karlskrona, Sweden. \\ \{wlodek.kulesza; ingvar.gustavsson; \\ kristian.nilsson\}@bth.se. \\ ${ }^{5}$ School of Engineering of Polytechnic \\ of Porto (ISEP). \\ Porto, Portugal. \\ \{anf; gca; mmr\}@ isep.ipp.pt. \\ ${ }^{6}$ University of Deusto. \\ Bibao, Spain. \\ \{unai.hernandez; zubia\}@ deusto.es.
}

\author{
Christian Kreiter ${ }^{7}$, Andreas Pester ${ }^{7}$, \\ Carla Garcia-Hernandez ${ }^{8}$, Ricardo \\ Tavio $^{8}$, Kati Valtonen ${ }^{9}$, Elina \\ Lehtikangas ${ }^{9}$ \\ ${ }^{7}$ Carinthia University of Applied \\ Sciences (CUAS). \\ Villach, Austria. \\ c.kreiter@fh-kaernten.at ; \\ pester@cti.ac.at. \\ ${ }^{8} \mathrm{EVM}$. \\ Tenerife, Spain. \\ \{carla.garcia; ricardo.tavio\}@evm.net. \\ ${ }^{9}$ OMNIA. \\ Helsinki, Finland. \\ \{kati.valtonen; \\ elina.lehtikangas\}@omnia.fi.
}

\begin{abstract}
Social demands have promoted an educational approach based on an "anywhere and anytime" premise. Remote laboratories have emerged as the answer to the demands of technical educational areas for adapting themselves to this scenario. The result has not only benefit distance learning students but has provided new learning scenarios both for teachers and students as well as allowing a flexible approach to experimental topics. However, as any other solution for providing practical scenarios (hands-on labs, virtual labs or simulators), remote labs face several constraints inherited from the subsystems of its deployment - hardware (real instruments, equipment and scenario) and software (analog/digital conversions, communications, workbenches, etc.)—. This paper describes the Erasmus+ project Platform Integration of Laboratories based on the Architecture of visiR (PILAR) which deals with several units of the federation installed in different educational institutions and devoted to analog electronics and electrical circuits. Based on the limitations of remote labs, the need for the federation will be justified and its benefits will be described by taking advantage of its strengths. The challenges that have come up during the different stages and the different approaches to design are also going to be described and analyzed.
\end{abstract}

Keywords-remote lab, VISIR, PILAR, electronics, federation, laboratory.

\section{INTRODUCTION}

Social demands have promoted an educational approach based on an "anywhere and anytime" premise. Based on this approach, educational institutions have fostered accessible educational resources through the Internet in order to enhance lifelong learning and support education trends (online, blended, distance learning, etc.). This new educational scenario is able to cover most areas of knowledge. However, most of the time practical training in technical courses is as important as theoretical concepts. Simulators are excellent tools that have been traditionally used in on-campus education, blended learning models, and, indeed, in distance education. Simulators are vastly used in educational institutions and provide remarkable benefits. Some of them are unique and unreachable from other options. But unfortunately to provide "laboratory experiences", simulators cannot fulfill a key feature only available in -and inherent to- real labs: working with real systems and real measurements. Regardless the importance of experimenting in the real world, simulators still play an important role in educational purposes.

Providing real experimental resources compatible with this new educational model is a challenge. Remote laboratories -a real laboratory (a system using real equipment, on which measurements are carried out through real instruments) which is remotely controllable - are the result of this interest in accessibility for technical courses.

In the field of analog electronics, several solutions have been developed to grant students access to remote experimentation [1]-[5]. These remote laboratories have different educational aims, and are reflected in their design, For instance, NetLab remote laboratory (University of South Australia, UniSA, Adelaide, Australia) is one of the pioneers (2002) in offering a system in which students are able to design their own circuits (Circuit Builder), as the components and instruments will be connected in the relay switching matrix [6]; eLab3D - deployed the in 3DLabs platform (Technical University of Madrid, UPM, Madrid, Spain)— is an analog electronics lab focusing on the immersive aspects of their 3D virtual world environment [2]; LaboREM (Bayonne Technological University Institute, Anglet, France) is an analog electronics lab working on active filters and where students can experiment with either predesigned circuits (named as DUTs, Devices Under Test) or build them with of a remotely controlled robotic arm and a webcam [7]; the Virtual Instruments System In Reality (VISIR) project started at the Department of Signal Processing of the Blekinge Institute of Technology (BTH) in 2006 [8]. The VISIR system replicates a hands-on analog electronics lab on a virtual workbench and it is based on a switching matrix relay for providing users a flexible experimental environment. 
Irrespective of the area of knowledge in which it is applied on, a remote lab is a combination of hardware -equipment, components, instruments, electrical and signal wiring, etc.and software [9]-[12] - Graphical User Interface (GUI), experiment protocol, client-server, equipment/instrument server, experiment server, etc.- Therefore, remote laboratories inherit the benefits of real systems and telecommunications in order to make possible a real system remotely to be accessible and controllable [13], [14], with the limitations and constraints associated to these two technologies (automation, time response, authentication, etc.). Consequently, there are strengths and weaknesses in these design decisions.

This paper describes Erasmus+ project PILAR (Platform Integration of Laboratories based on the Architecture of visiR) which deals with the advantages and disadvantages of remote laboratories (particularly the VISIR remote lab), the needs of the project, the cross-cutting benefits of its implementation, how the project empowers each node and how the goals have been faced and accomplished so far. Section II hereunder provides a detailed description of the VISIR remote lab and section III tackles all the aspects on the PILAR consortium.

\section{VISIR REMOTE LAB}

The flexibility and reliability of the VISIR remote lab have been reported numerous times [18]-[20]. It has been used in several learning scenarios [1], [19], different courses designs [21] and in secondary and higher education [22], vocational education [23], undergraduate [21], postgraduate [24], Massive Open Online Courses (MOOCs) [23], life-long learning[23], etc. Furthermore, in 2015 VISIR was awarded as the best remote laboratory by the Global Online Laboratory Consortium (GOLC) [18].

Another evidence of the reliability of the VISIR system is the number of nodes installed since its release. In 2016 at the beginning of the PILAR Erasmus+ project, there were $10 \mathrm{PXI}-$ VISIR systems - based on a PXI (PCI eXtensions for Instrumentation) instrumentation platform - and 1 LXI-VISIR system -based on a LXI (LAN eXtensions for Instrumentation) instrumentation platform developed at UDEUSTO- deployed in 8 different Higher Education Institutions (HEI) from 6 different countries: 2 in Sweden, both at BTH; 2 in Austria, at the University of Carinthia of Applied Sciences (CUAS) and at the FH Campus Wien for Applied Sciences; 3 in Spain, 2 at the University of Deusto (UDEUSTO), including the LXI system, and at the Spanish University for Distance Education (UNED); 2 in Portugal at the School of Engineering of the Polytechnic of Porto (ISEP); in Georgia at the Batumi Shota Rustaveli State University (BSU); and in India at the Madras Institute of Technology (MIT). 5 HEI from the aforementioned (BTH, CUAS; UDEUSTO; UNED and ISEP) are the VISIR initial nodes of the federation. In 2017 with the support of the Erasmus+ project "Educational Modules for Electric and Electronic Circuits Theory and Practice following an Enquiry-based Teaching and Learning Methodology supported by VISIR" (VISIR+ project 2015/2018) [26] and with the VISIR nodes from PILAR as partners, new VISIR systems have been installed in several South American HEIs: in Argentina at the National University of Rosario (UNR) and the National University of Santiago del
Estero (UNSE); and in Brazil at the Federal University of Santa Catarina (UFSC), the Federal Institute of Santa Catarina (IFSC) and the Pontifical Catholic University of Rio de Janeiro (PUC). Furthermore, ISEP has installed other VISIR system and new VISIR systems are being installed in North Africa and in Central America.

The VISIR remote lab, as previously commented, is built based on the development of 2 different elements to address remote control: hardware and software.

\section{A. Hardware}

Remote labs require that every experiment implemented must be designed to be reset automatically (without human intervention), instruments and equipment digitally accessible and either the experiment to be visually analyzed through a webcam or to require instruments which allow data acquisition streaming for monitoring the experiment. The hardware in VISIR remote lab can be clearly divided into two key elements: instrumentation platform and relay switching matrix. Communication between both subsystems is carried out through a USB cable.

1) Instrumentation platform: the instrumentation platform (Fig. 1) at least consists of a National Instrument (NI) chassis and the Peripheral Component Interconnect (PCI) eXtensions for Instrumentation (PXI) modules (function generator, DC power supply, oscilloscope and multimeter) and either an external PC or an embedded one as a system controller. housed in it. The chassis also provides a communication bus for the PXI instruments housed and power and cooling. The instrumentation platform shown in Fig. 1 is composed, from left to right, by an embedded controller, an oscilloscope, the power supply, the function generator and a digital multimeter.

2) Relay switching matrix: The relay switching matrix (Fig. 2) is a stack of two types of boards: component boards and instrument boards.

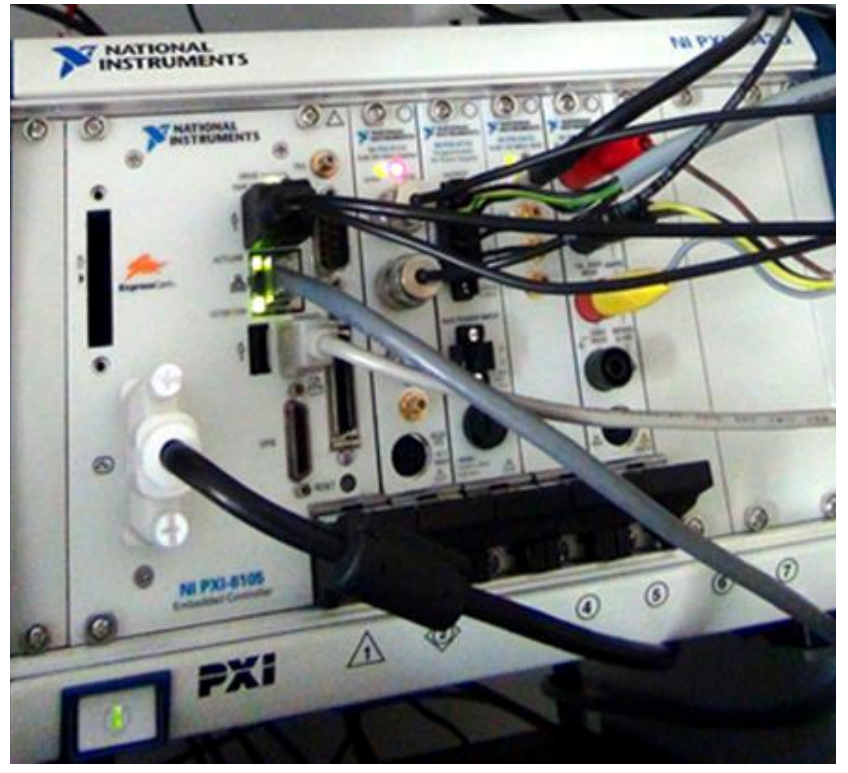

Fig. 1. PXI Chassis into which all the instrument cards (NI PXI modules) and the NI PXI controller are plugged, and relay switching matrix. VISIR system at UNED. 


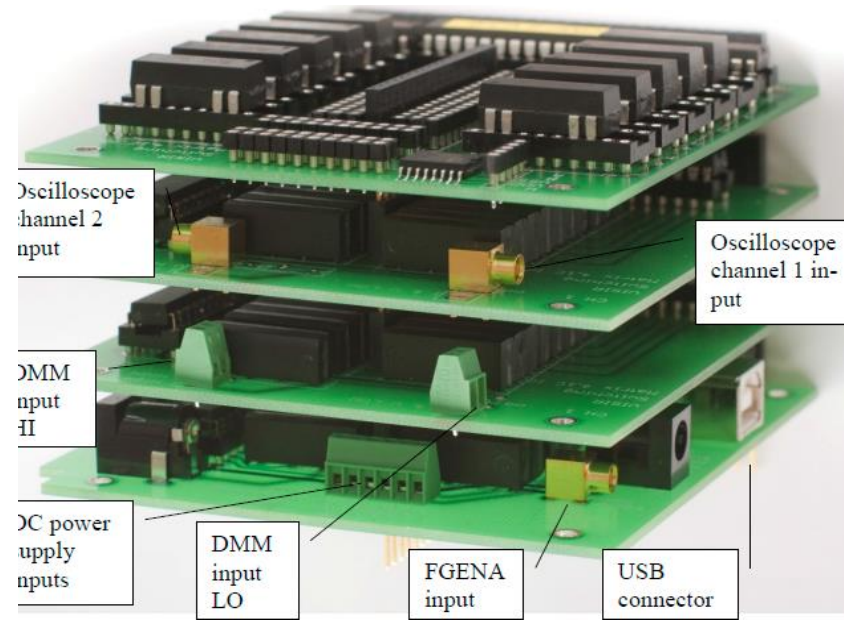

Fig. 2. Basic relay switching matrix [28]

a) Instrument boards are intended for the connection of the PXI instrument module cards to the matrix. For a typical electronics lab counting with function generator, DC power supply, oscilloscope and multimeter, 3 instrument cards are required.

b) Component boards accommodate the components available in the "VISIR component virtual store" for experimenting. There are 3 types of component boards:

- Component boards, ver.C: they accommodate ten dual port relays (Double Pole Single Throw, DPST). (20 leads). The connections to the 3D node matrix are rigid.

- Component boards, ver.D/E: their configuration is flexible as they can accommodate up to ten dual port relays, resulting in a configuration similar to the component board ver.C, but 6 of them can be replaced by single port relays resulting a board of 4 DIL relays and 12 SIL relays. The slots with SIL relays are intended specifically for components with more than two leads.

- Component boards $2 C$, ver.B: These component boards can only accommodate two two-leads components, but they connect each component lead with one of five nodes.

\section{B. Software}

The VISIR software is an open-source, released under GNU General Public License (GPL) [8]. It can be divided into client software, management software and experiment software. Hereunder we detail the main elements of each one.

1) Experiment Client: It is where the user interacts with the remote lab (GUI). The simulated workbench in VISIR is shown in Fig. 3. The students can switch between the different instruments to configure them, with, the component list available to students or not. If the access to the component list is denied, students can only experiment with the selected components at the component tray. Once a user clicks on the perform experiment button, the Experiment Client sends the experimentation requests to the Measurement Server using the Experiment Protocol -an XML-based protocol which describes the experiment designed by the user-. Once the system finds the response, the Experiment Client receives the information to be shown to the user in the same format in which the requests are sent (Experiment Protocol). The experiment Client handles only one request at a time.

2) Measurement Server: The Measurement Server, a software written in $\mathrm{C}++$, receives the requests from the Experiment Client; checks the authentication of the

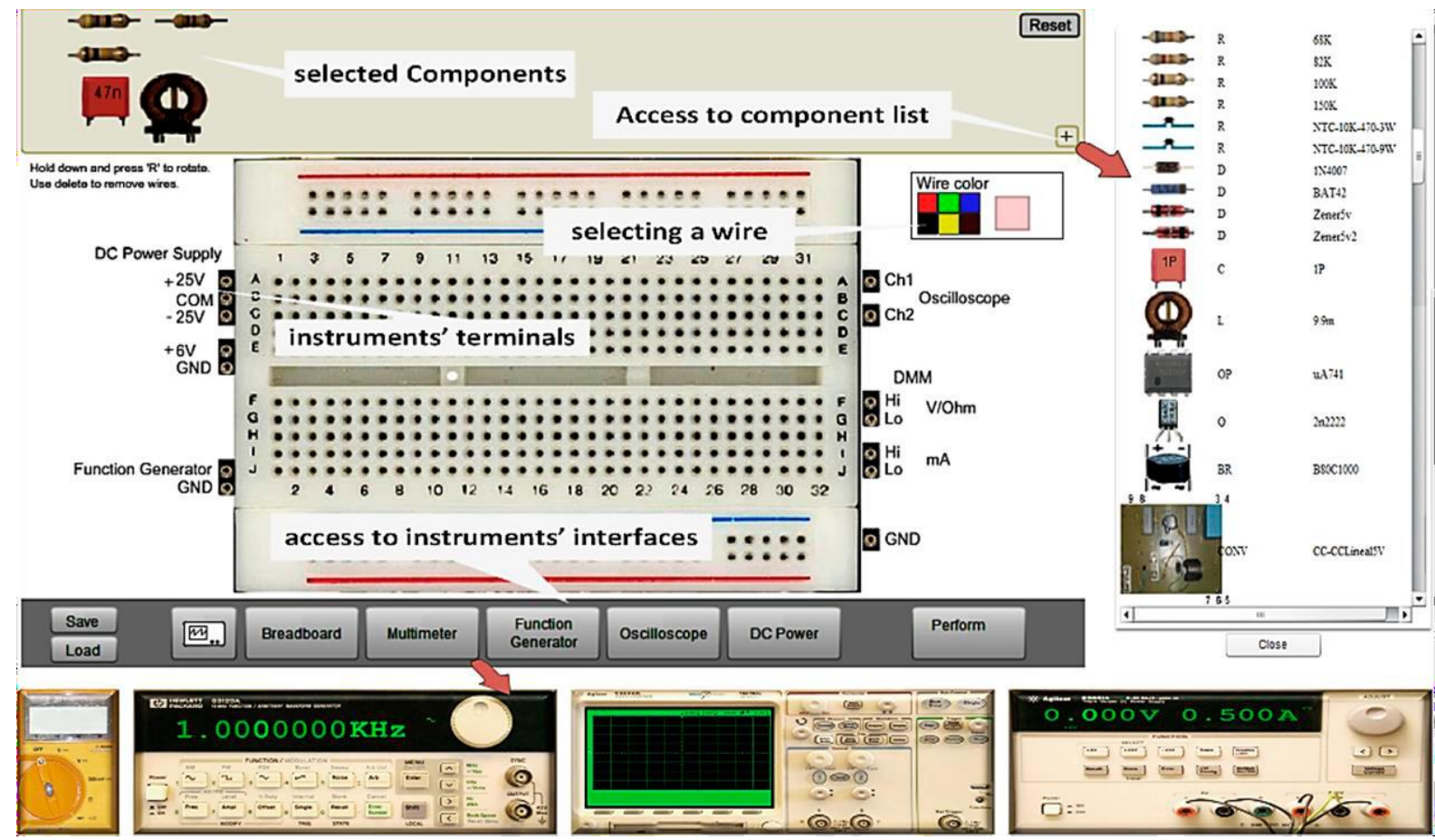

Fig. 3. VISIR simulated workbench. 
user;collates the designed experiment described in the Experiment Protocol with the max files repository - the max files describe the allowable experiments; queues the requests from concurrent users; sends the validated requests to the Equipment Server and provides the experiment responses to the users through the Experiment Client.

3) Equipment Server: The Equipment Server is the software, developed in LabVIEW, responsible for controlling the hardware of the VISIR system —instrument modules (directly connected to the relay switching matrix, Fig. 1 and Fig. 2) and relay switching matrix (through an USB cable)according to the validated requests of the users transferred from the Measurement Server.

\section{THE PILAR PROJECT}

The Erasmus+ PILAR project [27] proposes a solution that aims to interconnect all VISIR systems, with each other in order to create a grid laboratory shared and accessed by all the participants, expanding and empowering existing systems to a new level of service and capacity. The expected results are a well-defined set of open remote VISIR practices, available through Internet as a set of SLA-controlled services, for different subjects at grade and masters as well as high school levels. The project starts from 5 VISIR systems (BTH, CUAS, IPP, UDEUSTO and UNED) operating in isolated mode. These systems are going to be considered as nodes of a grid of shared experimental resources.

\section{A. Description}

The PILAR partnership is formed by 8 participants: 5 HEIs with extensive experience on undergraduate/postgraduate education and the integration of VISIR in engineering curricula (BTH, UNED, UDEUSTO, IPP and CUAS) where the VISIR nodes are located in; OMNIA, the Joint Authority of Education in the Region of Espoo, Finland, in charge of the training activities, a multi-sector education supplier of training workshops, non-formal education courses, vocational education and apprenticeship training for upper secondary education; EVM, a private organization which provides support to lead and coordinate the dissemination actions; and the International Association of Online Engineering (IAOE), a non-profit international organization focused on fostering online engineering technologies and its research in HEIs.

As aforementioned, VISIR has been proven as an effective tool for providing an anywhere and anytime electronics/electrical practical environment for students, teachers and researchers. This environment has been enriched by the continuous contribution of the VISIR community, especially by the VISIR special interest group (VISIR-SIG) [29]. PILAR evolves from this VISIR-SIG, by federating VISIR systems and sharing these resources available at each node in a more efficient way, leaving aside some of the existing limitations at each node and making this process transparent to the final user.

As an additional feature of PILAR, the VISIR systems federation through the PILAR consortium will be open to any institution interested in providing their students with the available resources.

\section{B. Goals in PILAR}

The VISIR federation is considered as the Intellectual Output 1 (IO1) of the project. The IO1 will be dynamically defined and built throughout the lifespan of the project (2016-2019). Its main objectives are:

- Building a reliable, highly available, unique international VISIR platform federation that integrates all the different resources used by VISIR in each of the partners. The details of courses and experiments provided by PILAR to each institution will also be described.

- $\quad$ Providing a set of remote practices for electrical and electronics circuits covering all the educational levels that are offered as remote lab services in all the partners' institution.

- Taking advantage of this new platform, to design and implement a completely new and innovative set of electrical and electronics circuits and the practical part of the subjects related to them.

- Allowing these new remote lab VISIR Internet services, the use, in a transparent way, of the best set of remote learning services of each partner in each moment.

- The federation is to be completely open to other partners, thus enabling to extend the capabilities of PILAR to other educational institutions interested in VISIR remote services. This step requires the creation of a set of formal documentation for institutions to join the PILAR consortium and the use of services by educational institutions.

- A much better control of the learning process of students when they carry out real practices on electrical and electronics experiments through the remote laboratories provided by PILAR.

\section{PILAR}

A VISIR system working in island mode provides obvious benefits to students whatever their needs are. The most obvious cases are those students with geographical restrictions that have not access to electronics hands-on labs or those students that due to life/job circumstances have temporal limitations. Nevertheless, students not belonging to these groups can be interested in extending experimentation scenarios and benefits beyond hands-on lab hours. However, a VISIR system working as an independent unit, although quite powerful, has limitations on the total number of components it can house and the available allowed interconnections. These limitations can be summarized as follows:

- Number of component boards: In [30] the authors describe these limitations. To summarize, all of them derive from the maximum number of components that a VISIR system can house. Each VISIR system can stack 15 components boards at most. Table I shows the number of boards operational at each node. The two numbers in the columns are related to each 
individual VISIR system for partners with two separate systems.

TABLE I. VISIR SYSTEM BOARDS BY INSTITUTION

\begin{tabular}{|c|c|c|c|}
\hline Partner & $\begin{array}{c}\text { Instrument } \\
\text { Boards }\end{array}$ & $\begin{array}{c}\text { Component } \\
\text { Boards }\end{array}$ & $\begin{array}{c}\text { New 2C } \\
\text { Boards }\end{array}$ \\
\hline BTH & $4 / 4$ & 6 & $2 / 2$ \\
\hline UNED & 4 & 10 & 0 \\
\hline CUAS & 3 & 6 & 0 \\
\hline UDeusto & $3 / 3$ & $8+6$ & 0 \\
\hline ISEP/IPP & $3 / 3$ & 4 & 3 \\
\hline
\end{tabular}

- Available components: Depending on the component board versions - component board version C (10 DIL relays), component board version D/E (6 DIL relays and 4 DIL relays that can be replaced by 2 SIL relays) and component board $2 \mathrm{C}$ - there is a limitation in the number of relays available for isolating/connecting components to the node matrix. Fig. 4 shows a component board version $\mathrm{E}$ in which the different configuration of the relays is shown. Table I details the type of component boards installed at each node.

- The limitation in the number of components available

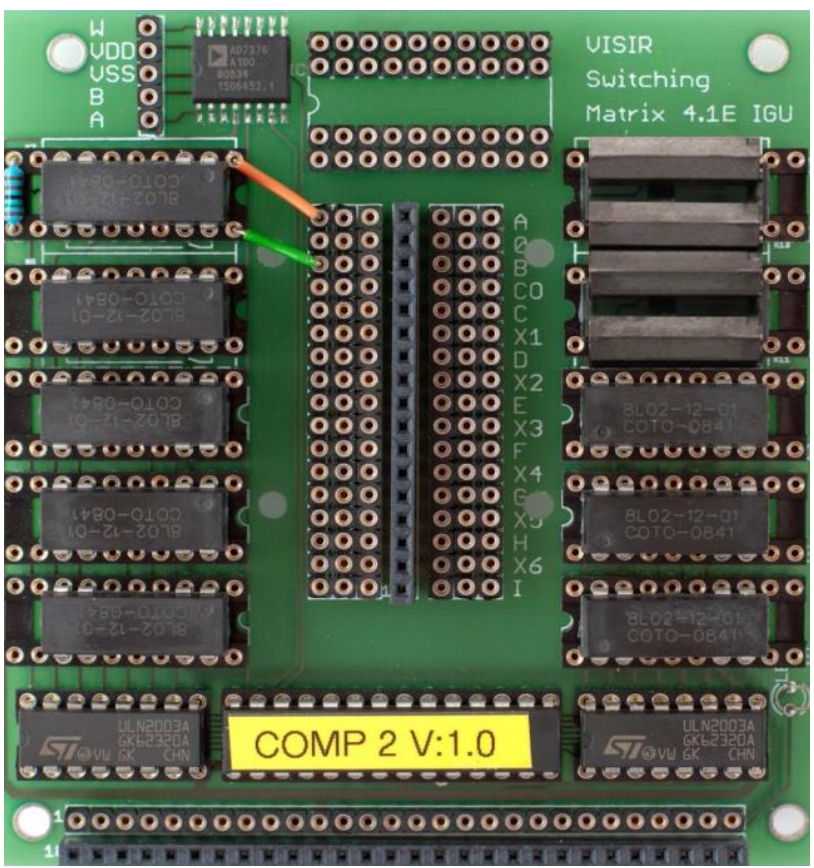

Fig. 4. Component board ver.E

in the switching relay matrix is not the only physical constraint of the system. In the aforementioned Fig. 4, the stack of component boards generates a 3D component matrix by means of the nodes propagating connector (in the center of the component boards). When using component boards $\mathrm{C}, \mathrm{D}$ or $\mathrm{E}$, once a component is wired to the matrix nodes of a board with a relay, it is fixedly wired to them. Fig. 4 shows a resistor wired to nodes $\mathrm{A}$ and $\mathrm{B}$ of the matrix nodes. Thus, a switching relay matrix can house a set of components but it does not mean that any design can

$\begin{array}{llll}\text { VFGENA_FGENA1 } & \text { A } 0 & \max : 5 & \\ \text { VDC+25V_4 } & \text { B } & \max : 15 & \text { imax:0.5 } \\ \text { VDC-25V_5 } & \text { C } & \begin{array}{l}\max :-15 \\ \text { imax:0.5 } \\ \text { VDCCOM_24_2 }\end{array} & \\ & & \\ \text { R_3_6 H I 100K } & & \\ \text { R_5_2 H I 47K5 } & & \\ \text { R_9_2 H I 20K } & \\ \text { R_7_3 H I 10K } & \\ \text { SHORTCUT_8_9 E H } & \\ \text { SHORTCUT_8_8 G 0 } & \\ \text { OP_5_4:5_5:5_6:5_7:1_11 NC1 H G C NC2 I B NC3 UA741 } \\ \text { SHORTCUT_6_12 A F } \\ \text { R_2_9 E F 10K }\end{array}$

Fig. 5. *.max file describing a subset the available components in the relay switching matrix. The referenced components and shorcircuits must be physically implemented in the switching relay matrix.

be implemented with them. Component boards $2 \mathrm{C}$ are flexible in terms of which nodes are used, but they only can house 2 2-leads components. Fig. 5 contains the description of an operational amplifier as an inverter. There are 4 resistors connected between $\mathrm{H}$ and I nodes in order to vary the amplification level. This experiment requires, at least, 5 DIL relays and 5 SIL relays. To summarize, in [30] authors highlight the need for a federation due to the difference between a switching relay matrix typical storage capacity and a typical electronic components store.

- Concurrent users: The number of feasible concurrent users, experimenting with the same or different components and/or instruments, supports the potential of a federation. No more than 60 students can experiment with a VISIR system. However, as described in [31], a high number of concurrent users managed by a VISIR system causes delayed system responses when managing the requests and, consequently, a bad user experience in the platform. Thus, concurrent users in a VISIR system must be more restrictive than 60 .

\section{Further steps in the PILAR federation}

In the literature there are different technologies available that act as software to manage remote laboratories and are described as Remote Laboratory Management Systems (RLMS). Examples of these are the MIT iLabs system [32], WebLab-Deusto [33], RemLabNet [34] or Labshare Sahara [35].

These technologies provide common features of remote laboratories, such as authentication, authorization, scheduling, user administration or even learning analytics. VISIR comes with its own built-for management tools (called OpenLabs), but the lack the integration in external tools that the others provide. The key feature of the RLMS is that if third parties build on top of an RLMS, this can automatically be applied on all the laboratories that are supported by this RLMS. For example, any new feature on WebLab-Deusto will be available in all the VISIR systems in the different institutions using VISIR.

In a first step, each practical resource has relied on a specific VISIR node. Each practical experience is rigidly linked to a specific node; i.e., a practical experience replicated in different nodes can coexist but, initially, the platform considers both as different experiments. Final users do not need to be aware of which VISIR node they are accessing to. This first approach has fulfilled the following requirements: 
- FA1. Enable the access of teachers and students to all the VISIR systems of PILAR.

- FA2. The solution must be federated, meaning that teachers and students will not need to be registered in each of the VISIR systems of the PILAR consortium.

- $\quad$ FA3. Students and teachers do not need to be aware of which VISIR system they are using. They should be able to move from one system to other without knowing what VISIR system supports what.

- $\quad$ FA4. Teachers must be able to try the lessons without having their own infrastructure.

- $\quad$ FA5. Teachers must be able to use the contents without having their own infrastructure.

At the end of this phase, although the users do not need to be aware of which VISIR system they are using, the system does not have control on the selection of node made by the user. A practical experience replicated in different nodes can coexist but the node in which the experimentation is carried out is chosen by the PILAR user. This is reflected in Fig. 6, where three users (user_a, user_b and user_c) are accessing to a replicated experiment (exp1) in 2 nodes (CUAS and UNED). As the system does not have control over the selection of node, it can happen that all users decide to access the same node, a problem that we have overcome in this stage.

In next phase final users do not need to be aware of which VISIR node they are accessing to. Subsequently, the system is able to balance the users load accessing the same resource between the nodes which provide it. This balance should be transparent for the final users. At the end of this phase the following repositories will be covered in future versions of the lesson repository of PILAR:

- FR1: Support of distributed load balancing: if a circuit is available in more than one VISIR system, students should be dynamically redirected to one or the other
- FR2: Teachers and students must be able to count with contents in multiple languages.

- $\quad$ FR3: Teachers must be able to have a single repository to search for lessons and contents.

- $\quad$ FR4: Teachers must be able to take the contents and lessons and bring them to their own spaces outside PILAR by using an assistant.

- FR5: The administrative layer in PILAR will be in charge of checking the node status before providing the service to users.

At the end of this phase the system will balance the users load between the nodes which provide the required service, as shown in Fig. 7 users will access to a generic experiment or service (exp1, exp2, etc.) and the system will be in charge of sending the user to the appropriate node and, in case of a resource replicated in several nodes, it will balance the users load. This phase should be completed at the end of 2018 .

\section{E. Architecture}

PILAR resources are deployed and available in a Moodle platform. As a first step, it is possible to integrate all the laboratories using WebLab-Deusto. Using its federation properties, it is possible to have a second WebLab-Deusto system that interacts with each of the WebLab-Deusto systems, thus providing a single interface for all the VISIR systems.

Four nodes have been fully integrated into the system: UDEUSTO and IPP through their own WebLab-Deusto deployment, and BTH and CUAS nodes through a WebLabDeusto deployment dedicated to the PILAR consortium. The fifth one - the UNED node although it is integrated into its own WebLab-Deusto deployment, it requires a different approach: the practices and circuits designed at the UNED node and the resulting needs in using a booking system discerns between the availability of the VISIR lab in concurrent or private mode.

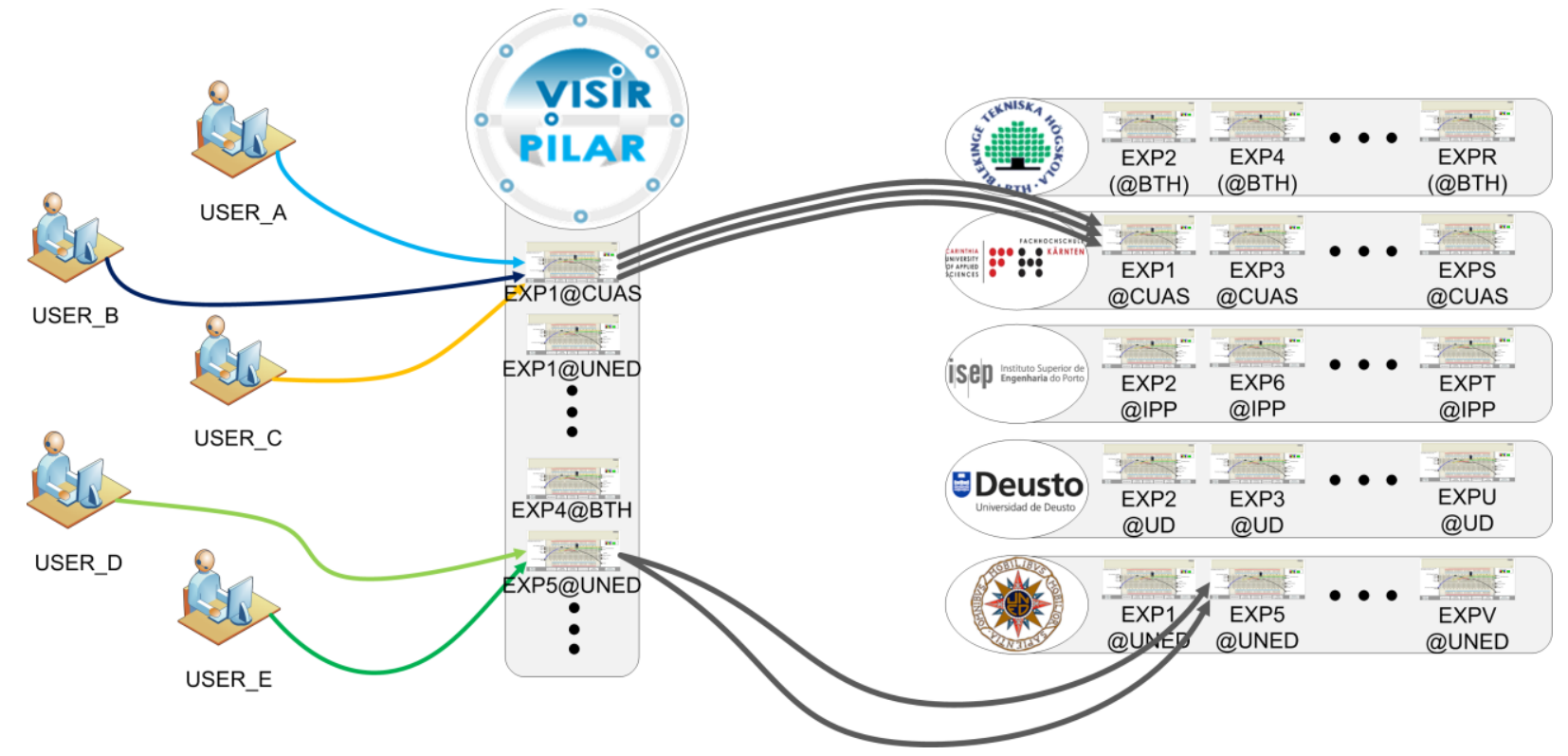

Fig. 6. PILAR at first stage. 


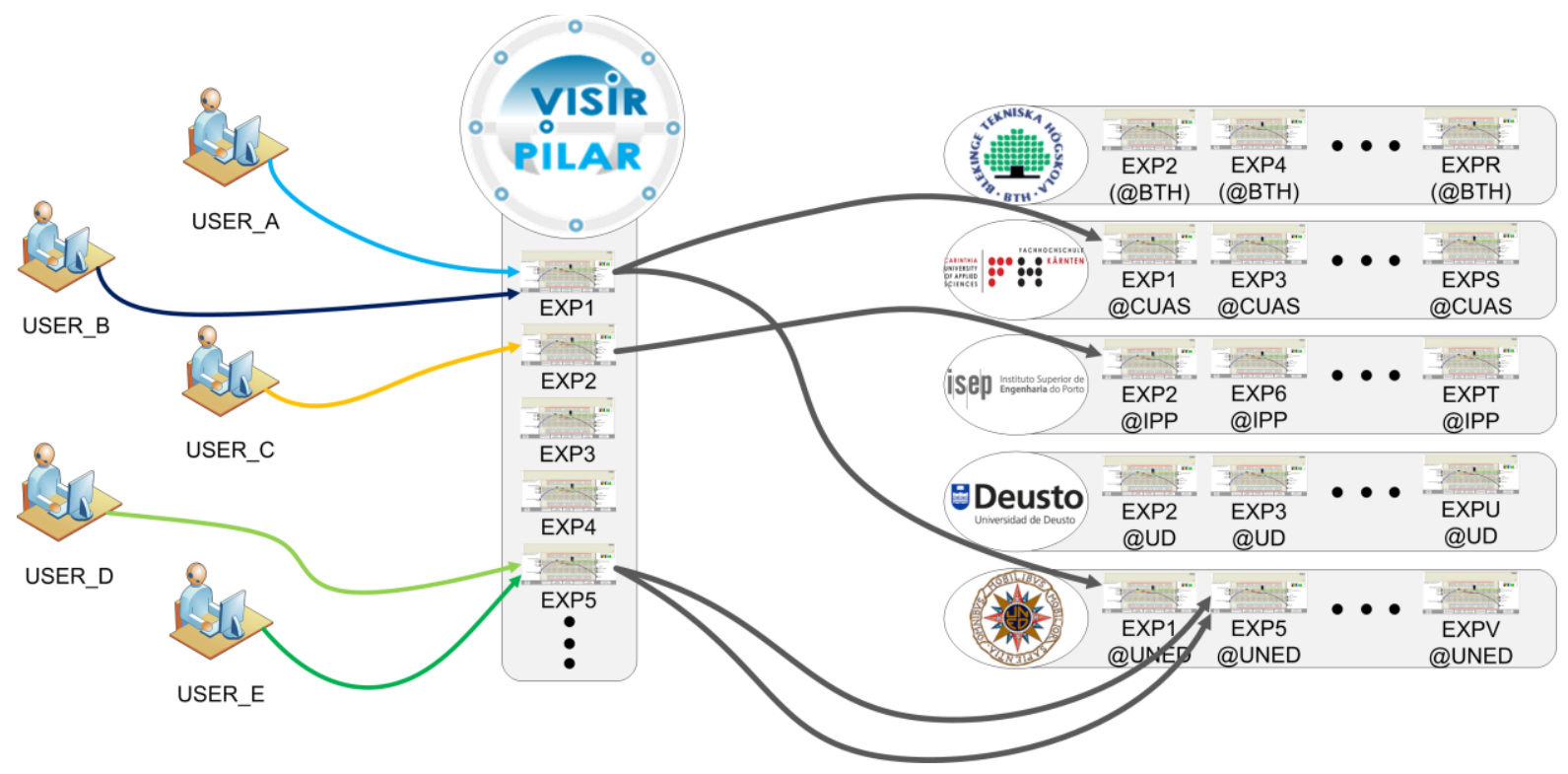

Fig. 7. PILAR at second stage.

\section{CONCLUSIONS AND FUTURE WORK}

In educational contexts the term federation has been traditionally used for describing a common platform containing educational resources that can be shared by different institutions. The use of a federation intended for the PILAR project must be designed aiming at not only to foster the diversity in the number of practical experiences but to empower each node in terms of reliability and availability.

At the end of the first stage of the federation the following benefits have been achieved:

- Each node has empowered the reliability of the 24/7 availability by sharing practical resources.

- Each node has broadened its resource repository by sharing practical resources.

- PILAR provides real, extensive and intensive, online, cheap practices for building and interacting with electrical and electronics circuits.

- A reliable, highly available, unique international VISIR platform federation that integrates all the different resources used by VISIR in each of the partners has been established.

In summary PILAR has improved the efficiency of the already existing VISIR systems and nowadays it is providing an extensive resource repository to any educational institution interested in VISIR services.

The reliability of a VISIR system working as an isolated unit has been broadly described by the five nodes used in the project. However, in the short term a series of features must be implemented in order to empower the reliability and availability of the federation. A script which systematically sends requests to each node must be used for identifying if the lab services are available. This VISIR availability service within PILAR should be used, ideally, every time a PILAR user tries to access a practice lab service. The system must also be able to balance the users load between the nodes which provide the required service at each moment.

The implemented PILAR system is already a tool which can assist in increasing the number of graduates in the university. However, the second phase will empower and increase the benefits provided in this first stage.

Nevertheless, a much better control of the learning process of the students when they carry out real experiments through the VISIR systems provided by PILAR is now possible due to the architecture implemented.

In the short term, this new platform and federation allows to design a much larger set of new and innovative practices, oriented to different and updated needs for students in different academic levels.

\section{ACKNOWLEDGMENT}

The authors acknowledge the support of the Escuela de Doctorado de la UNED, the S2013/ICE-2715 - eMadrid project, VISIR+ project Erasmus+ Capacity Building in Higher Education $2015 \quad \mathrm{n}^{\circ}$ 561735-EPP-1-2015-1-PT-EPPKA2CBHE-JP and PILAR project Erasmus+ Strategic Partnership $\mathrm{n}^{\mathrm{o}}$ 2016-1-ES01-KA203-025327 (Platform Integration of Laboratories based on the Architecture of visiR). As well as to the Education Innovation Project (PIE) of UNED, "Desarrollos Avanzados Multi-Objetivo de Laboratorios Remotos para Actividades Educativas - DAMO-LRAE", from the Vicerrectorado de Ordenación Académica y Calidad and the Instituto Universitario de Educación a Distancia (IUED) of the UNED and to the project 2018-IEQ18 from the Escuela Superior de Ingenieros Industriales of UNED.

\section{REFERENCES}

[1] A.V. Fidalgo, G.R. Alves, M.A. Marques, M.C. Viegas, M.C. CostaLobo, U. Henandez-Jayo, J. Garcia-Zúbia and I. Gustavsson, '"Adapting Remote Labs to Learning Scenarios: Case Studies Using VISIR and 
RemotElectLab," IEEE Revista Iberoamericana de Tecnologias del Aprendizaje, vol. 9, no. 1, February, pp. 33-39.

[2] S. López, A. Carpeño and J. Arriaga, "Remote Laboratory eLab3D: A Complementary Resource in Engineering Education," IEEE Revista Iberoamericana De Tecnologias Del Aprendizaje, vol. 10, (3), pp. 160 167, 2015. . DOI: 10.1109/RITA.2015.2452711.

[3] Z. Nedic, J. Machotka and A. Nafalski, '"Remote laboratories versus virtual and real laboratories," 33rd Annual Frontiers in Education, 2003. FIE 2003., vol. 1, pp. 6 Vol.1.

[4] D.A.H. Samuelsen and O.H. Graven, '"Design of a general purpose platform for easy setup of low-cost remote laboratories in electronics," REV, pp. 1-6.

[5] F. Hobar and L. Semra, "'Development and implementation of an ecourse and a remote laboratory for analog electronics study,", 2014.

[6] M. Stefanovic et al, "An assessment of distance learning laboratory objectives for control engineering education," Computer Applications in Engineering Education, vol. 23, (2), pp. 191-202, 2015. Available: https://onlinelibrary.wiley.com/doi/abs/10.1002/cae.21589. DOI: $10.1002 /$ cae.21589.

[7] F. Luthon and B. Larroque, "LaboREM-A Remote Laboratory for GameLike Training in Electronics," IEEE Trans. Learn. Technol., vol. 8, (3), pp. 311-321, 2015. . DOI: 10.1109/TLT.2014.2386337.

[8] I. Gustavsson, J. Zackrisson, L. Håkansson, I. Claesson, T. Lagö, "The VISIR project - an open source software initiative for distributed online laboratories," International Conference on Remote Engineering and Virtual Instrumentation (REV), 2007, Porto.

[9] BioLab, "BioLab | Transformative Learning Technologies Lab", Tltl.stanford.edu, $2018 . \quad$ [Online]. Available: https://tltl.stanford.edu/project/biolab. [Accessed: 04- Jun- 2018].

[10] NANSLO, "NANSLO Lab Activities | Western Interstate Commission for Higher Education", Wiche.edu, 2018. [Online]. Available: http://wiche.edu/nanslo/lab-activities. [Accessed: 04- Jun- 2018].

[11] WebLab-Deusto, "WebLab-Deusto | Labs", Weblab.deusto.es, 2018 [Online]. Available: https://weblab.deusto.es/weblab. [Accessed: 04Jun- 2018].

[12] iSES, "iSES - Internet School Experimental System: iSES Remote Lab SDK", Ises.info, 2018. [Online]. Available: http://www.ises.info/index.php/en/systemises/sdkisesstudio. [Accessed: 05- Jun- 2018].

[13] T. Mohamed, M. Santiago, Garcia - Loro Felix, S. Elio, R. Elena, Díaz Gabriel, S.A. Colmenar, P. Juan and C. Manuel, '"Novel design and development of advanced remote electronics experiments, "Comput Appl Eng Educ, vol. 23, no. 3, pp. 327-336.

[14] S. M. Gutierrez, L. J. Guirado and M. A. C. Gil, "Architecture of an Open Remote Lab for Digital Electronics," Dyna, vol. 91, (6), pp. 599600, 2016. Available: https://www.revistadyna.com/search/architectureof-an-open-remote-lab-for-digital-electronics.

[15] Lopez, S., Carpeño, A. and Arriaga, J., "Remote Laboratory eLab3D: A Complementary Resource in Engineering Education,", IEEE Revista Iberoamericana de Tecnologías del Aprendizaje, 10(3), pp.160-167, 2015.

[16] M. Tawfik et al, "Online Experiments With DC/DC Converters Using the VISIR Remote Laboratory," IEEE Rev. Iberoam. Tecnol Aprendiz., vol. 10, (4), pp. 310-318, 2015. DOI: 10.1109/RITA.2015.2486459.

[17] Z. Nedic, J. Machotka and A. Nafalski, "Remote laboratory netlab for effective interaction with real equipment over the internet," in May 2008,. DOI: 10.1109/HSI.2008.4581553.

[18] I. Gustavsson, K. Nilsson, J. Zackrisson, G.R. Alves, A.V. Fidalgo, L. Claesson, J.G. Zubia, U.H. Jayo, M. Castro, G. Diaz Orueta and F.G. Loro, "'Lab sessions in VISIR laboratories," 2016 13th International Conference on Remote Engineering and Virtual Instrumentation (REV), pp. 350-352.

[19] M.I. Pozzo, E. Dobboletta, F. Garcia-Loro, E. Sancristoba, G. Diaz, M Castro, A. Fidalgo and G. Alves, '"Sharing educational experiences from in-person classroom to collaborative lab environments," 2017 IEEE Global Engineering Education Conference (EDUCON), pp. 1506-1512.
[20] I. Gustavsson, K. Nilsson, J. Zackrisson, J. Garcia-Zubia, U. HernandezJayo, A. Nafalski, Z. Nedic, O. Gol, J. Machotka, M.I. Pettersson, T. Lago and L. Hakansson, '"On Objectives of Instructional Laboratories, Individual Assessment, and Use of Collaborative Remote Laboratories," IEEE Transactions on Learning Technologies, vol. 2, no. 4, pp. 263-274.

[21] G.R. Alves, M.C. Viegas, M.A. Marques, M.C. Costa-Lobo, A.A. Silva F. Formanski and J.B. Silva, "'Student performance analysis under different moodle course designs," 2012 15th International Conference on Interactive Collaborative Learning (ICL), pp. 1-5.

[22] O. Dziabenko, P. Orduña and J. Garcia-Zubia, '"Remote experiments in secondary school education," 2013 IEEE Frontiers in Education Conference (FIE), pp. 1760-1764.

[23] F. Garcia-Loro, R. Fernandez, M. Gomez, H. Paz, F. Soria, M.I. Pozzo, E. Dobboletta, A. Fidalgo, G. Alves, E. Sancristobal, G. Diaz and M. Castro, '"Educational Scenarios Using Remote Laboratory VISIR for Electrical/Electronic Experimentation,", pp. 298-303.

[24] M. Tawfik, S. Monteso, F.G. Loro, E. Sancristobal, F. Mur, G. Diaz and M. Castro, '"Design of electronics circuits practices for an online master degree program using VISIR," 2013 IEEE Global Engineering Education Conference (EDUCON), pp. 1222-1227.

[25] F. Garcia, G. Diaz, M. Tawfik, S. Martin, E. Sancristobal and M. Castro, "'A practice-based MOOC for learning electronics," 2014 IEEE Global Engineering Education Conference (EDUCON), pp. 969-974.

[26] EC VISIR+, "Erasmus+ Project - Educational Modules for Electric and Electronic Circuits Theory and Practice following an Enquiry-based Teaching and Learning Methodology supported by VISIR", ec.europa.eu, 2018, [online] Available at: http://ec.europa.eu/programmes/erasmusplus/projects/eplus-project-details/\#project/5baadcef-0a61-4592-8196c63d200f36ea [Accessed 22-05-2018].

[27] EC PILAR, "Erasmus+ Project - Platform Integration of Laboratories based on the Architecture of visiR", ec.europa.eu, 2018 [online] Available at: http://ec.europa.eu/programmes/erasmusplus/projects/eplus-project-detailspage/?nodeRef=workspace://SpacesStore/2d88ecb1-3db1-4a29-93c1 dd2802eec4f6 [Accessed 22-05-2018]

[28] I. Gustavsson, "Visir Relay Switching Matrix Version 4.1 - User's Manual,", 2016.

[29] IAOE-GOLC, "International Association of Online Engineering (IAOE) \& Global Online Consortium (GOLC)", 2018, [online] Available at: http://online-engineering.org/ [Accessed 23-05-2018].

[30] F. Garcia-Loro et al, '"Experimenting in PILAR federation: A common path for the future," 2018 IEEE Global Engineering Education Conference (EDUCON), pp. 1518-1523.

[31] G.R. Alves et al., '"Spreading remote lab usage a system - A community - A Federation," 2016 2nd International Conference of the Portuguese Society for Engineering Education (CISPEE), pp. 1-7.

[32] V.J. Harward, J.A. del Alamo, S.R. Lerman, P.H. Bailey, J. Carpenter, K. DeLong, C. Felknor, J. Hardison, B. Harrison, I. Jabbour, P.D. Long, T. Mao, L. Naamani, J. Northridge, M. Schulz, D. Talavera, C. Varadharajan, S. Wang, K. Yehia, R. Zbib and D. Zych, "'The iLab Shared Architecture: A Web Services Infrastructure to Build Communities of Internet Accessible Laboratories," Proceedings of the IEEE, vol. 96, no. 6, pp. 931-950.

[33] P. Orduña, J. Irurzun, L. Rodriguez-Gil, J. Garcia-Zubia, F. Gazzola and D. López-de-Ipiña, '"Adding New Features to New and Existing Remote Experiments through their Integration in WebLab-Deusto," International Journal of Online Engineering (iJOE), vol. 7, no. S2, /10/15, pp. 33-39.

[34] F. Schauer, M. Krbecek, P. Beno, M. Gerza, L. Palka and P. SpilakovÃ "'REMLABNET - open remote laboratory management system for $e$ experiments," 2014 11th International Conference on Remote Engineering and Virtual Instrumentation (REV), pp. 268-273.

[35] M. Diponio, D. Lowe and M. de la Villefromoy, '"Supporting local access to collections of distributed remote laboratories," 23rd Annual Conference of the Australasian Association for Engineering Education 2012: Profession of Engineering Education: Advancing Teaching, Research and Careers, The, pp. 968. 